Service social

\title{
Le tabou d'incentre ou la politique de l'interdit sexuel dans un centre hospitalier de soins prolongés.
}

\section{André Dupras}

Volume 35, numéro 3, 1986

Les jeunes et le travail social

URI : https://id.erudit.org/iderudit/706324ar

DOI : https://doi.org/10.7202/706324ar

Aller au sommaire du numéro

Éditeur(s)

École de service social de l'Université Laval

ISSN

1708-1734 (numérique)

Découvrir la revue

Citer ce document

Dupras, A. (1986). Le tabou d'incentre ou la politique de l'interdit sexuel dans un centre hospitalier de soins prolongés. Service social, 35(3), 457-474.

https://doi.org/10.7202/706324ar
Résumé de l'article

Un comité d'étude, mis sur pied dans un centre hospitalier de soins prolongés, a interrogé vingt-cinq personnes, membres du personnel, afin de connaître leurs représentations de leur milieu de travail et de la sexualité telle qu'elle y est vécue.

Amorcée dans le but de dégager la politique de l'établissement relativement aux questions sexuelles, cette analyse permet de conclure que l'expression de la sexualité est interdite (tabou d'/ncenfre).

L'auteur conclut en souhaitant que s'amorce un dialogue entre employé(e)s et bénéficiaires sur ce sujet et que l'établissement élabore une politique qui tienne compte de la force positive de la sexualité. 


\section{Le tabou d'incentre ou la politique de l'interdit sexuel dans un centre hospitalier de soins prolongés}

\section{André Dupras}

Dans les centres hospitaliers de soins prolongés, il arrive que des bénéficiaires expriment leur sexualité d'une manière jugée non acceptable par le personnel infirmier : par exemple, des patients de sexe masculin parlent de questions sexuelles d'une façon grivoise, font des attouchements sexuels sur eux-mêmes ou sur les autres, regardent des documents érotiques. Dans une étude réalisée dans un service de soins prolongés, Szasz (1983) a constaté que ces comportements étaient perçus comme une source de problèmes par le personnel infirmier. Par contre, donner un baiser, se tenir la main et s'entrelacer constituaient des gestes acceptables.

Les réactions du personnel infirmier aux comportements sexuels non acceptables sont variées et vont de l'indifférence à la punition, selon la gravité de l'acte et celui qui le constate. Lorsque le personnel infirmier s'interroge, dans des rencontres informelles ou lors de réunions, sur la meilleure attitude à prendre dans ces situations, on suggère habituellement de former un comité qui étudiera la question et proposera des modalités d'action. Après avoir obtenu des avis et consulté des ouvrages sur la sexologie (Cornelius et al., 1979; Solnik, 1978; Weg, 1983), le comité peut se donner comme premier mandat d'élaborer une politique institutionnelle à l'égard de la sexualité.

La politique institutionnelle constitue une ligne directrice relative à la sexualité, qui est établie par la direction de l'établissement et qui sera suivie par les personnes y œuvrant ou y résidant. Tous les acteurs du système institutionnel confrontés à des situations ayant un contenu sexuel doivent la suivre. La proposition de politique, élaborée par le comité, contient généralement un rappel des droits et des responsabilités de chacun, une présentation des objectifs que cherchent à réaliser les 
programmes d'activités et une réglementation (Hall, 1978; Keitner et Grof, 1981; Striar et al., 1981).

La popularité de cette dernière auprès des professionnel(le)s est sûrement attribuable aux avantages qui y sont rattachés : elle permet de définir la discipline à suivre à l'intérieur de l'établissement afin d'assurer son bon fonctionnement; elle donne l'impression qu'une pensée et qu'une action dorénavant concertées vont contribuer au bien-être sexuel des bénéficiaires et que l'utilisation commune de repères fiables et sûrs garantiront l'efficacité de l'intervention; elle offre la promesse d'un équilibre, tant pour l'individu que pour la collectivité.

Le recours à l'élaboration d'une politique institutionnelle représente souvent une stratégie pour apaiser des conflits suscités par l'émergence de problèmes reliés à la sexualité à l'intérieur de l'établissement. Les comportements sexuels inacceptables des bénéficiaires dérangent le personnel et le font se questionner sur les normes et les valeurs sociales relatives à la sexualité. En se réfugiant dans les règles normatives, il prévient ou désamorce les crises institutionnelles, tout en évitant de discuter des situations conflictuelles. Guidée par un souci d'efficacité et de paix sociale, la direction de l'établissement sera tentée d'édicter et d'implanter une politique pour améliorer les relations interpersonnelles et maintenir le système institutionnel en place. On peut facilement deviner les inconvénients de cette approche normative, qui ne règle pas toutes les insatisfactions et les malaises. Les tensions suscitées par l'expression manifeste de la sexualité ne sont pas totalement évacuées et risquent en tout temps de fissurer la sociabilité institutionnelle. Le processus d'élaboration d'une politique arrive rarement à mettre en relief les dimensions sous-jacentes du dynamisme de la vie institutionnelle concernant la sexualité. Pour le faire, il faut procéder autrement, en laissant les membres du personnel s'exprimer et en analysant leur discours sur la sexualité.

C'est ce qu'a entrepris un comité d'un centre hospitalier de soins prolongés. Il a convenu de recueillir les propos d'un groupe de professionnels afin de dégager le mode de fonctionnement, actuel et souhaité, en matière de sexualité au sein de l'établissement. Plus précisément, il désirait étudier la représentation de la sexualité des membres du personnel afin de mieux comprendre l'élaboration de leurs conceptions relatives à la sexualité et leurs orientations par rapport à celle-ci. Par le biais de ces représentations, ce sont les valeurs, les normes sociales et les modes culturels pensés et vécus par des personnes vivant dans ce milieu qui étaient examinés. On entend ici par représentation collective de la sexualité, l'élaboration psychologique complexe où s'intègrent une image signifiante de la sexualité, l'expérience de chacun(e), les valeurs et les informations circulant dans l'établissement. 


\section{Méthodologie}

L'orientation de l'étude nous obligeait à accorder au sujet une certaine liberté d'expression puisque ses propos constituent l'objet même de l'observation. Les données recueillies l'ont été par entretien semi-dirigé, l'entrevue encourageant le sujet à expliciter, sous diverses formes (opinions, informations, sentiments, récits d'expériences et comportements), son image de la sexualité. Au cours de la phase préparatoire à la recherche, une série d'entretiens a permis de sélectionner des thèmes pertinents par rapport à la population étudiée : la description du milieu hospitalier, la façon dont on parle de la sexualité, les limites imposées à l'intimité et le contrôle des comportements sexuels. Puisque l'objectif principal consistait à mettre à jour la problématique propre à chacun, les différents thèmes de l'entretien n'étaient pas ordonnés ou formulés de la même façon. L'entrevue durait en moyenne une heure et demie.

Le centre hospitalier où s'est déroulée l'étude regroupe 260 patients de sexe masculin et un personnel de $\mathbf{5 0 0}$ personnes. Les bénéficiaires ont de 19 à 97 ans, l'âge moyen se situant à 60 ans. Leurs troubles physiques sont dus à des accidents, ou, le plus souvent, à des maladies telles que la sclérose en plaques, le diabète et l'artériosclérose. Les handicaps les plus fréquemment rencontrés sont l'hémiplégie, la paralysie cérébrale et la paraplégie.

L'objectif de l'étude et le choix d'une approche qualitative commandaient la recherche de valeurs et de comportements relatifs à la sexualité les plus diversifiés possible. Il fallait donc solliciter la collaboration de membres du personnel représentatifs et variés; ainsi, vingt-cinq personnes ont été interrogées; l'âge moyen du groupe était de 37 ans et il comprenait quatorze femmes et onze hommes.

\section{Analyse des discours}

\section{Le milieu de travail}

Afin de situer le contexte dans lequel s'exprime la sexualité des bénéficiaires, nous avons demandé aux membres du personnel de nous parler de leur milieu de travail : décrire l'atmosphère de l'hôpital et les services qu'ils dispensaient. De ces propos, nous avons dégagé deux représentations du centre hospitalier : une famille et une prison. 


\section{L'hôpital : une famille}

Quand ils parlent de leur milieu de travail, les membres du personnel interviewés évitent de le décrire comme une maison de santé. L'établissement de soins prolongés reçoit des bénéficiaires qui côtoieront fréquemment et longuement les autres patients et le personnel soignant. Dans ce contexte, il ressemble plus à une maisonnée où des personnes habitent un même endroit et vivent ensemble. L'hôpital de soins prolongés devient un domicile ; on s'y établit, pour y vivre et mourir. On le décrit comme leur dernière demeure terrestre.

Les membres du personnel rencontrés ne considèrent pas les résidents comme des étrangers de passage mais comme des domiciliés, des membres de la maison qui participent à la vie domestique de l'établissement. Dans cette maisonnée, on retrouve des échanges et des relations qui ressemblent à celles qu'entretiennent les membres d'une famille. Aussi, on se côtoie assidûment, on apprend à se connaître, on se lie d'amitié avec certaines personnes et on devient plus intimes avec elles. La maison devient un lieu où les marques d'affection font partie des échanges quotidiens ; elles permettent de témoigner l'attachement des uns aux autres en plus d'entretenir l'atmosphère familiale.

D'après le personnel interviewé, l'établissement doit d'abord répondre aux besoins de base (soins médicaux et hébergement); il semble que les ressources humaines et physiques comblent adéquatement les besoins physiques; mais il doit également se préoccuper des besoins psychologiques et affectifs. Dans le contexte d'une hospitalisation à long terme, la santé mentale des bénéficiaires paraît sérieusement menacée : ils sont déracinés de leur milieu de vie habituel et souvent délaissés par leurs proches. Les liens d'amitié et d'amour deviennent ténus et sont parfois brisés définitivement. Leur carence affective se manifeste alors par une recherche de contacts physiques. Ils veulent être touchés pour sortir de leur solitude et pouvoir répondre à une manifestation d'attention à leur égard. Le toucher, le sourire et la parole sont des démonstrations d'affection, de bienveillance et d'amitié. À l'approche froide et sèche de certains employés, ils privilégient la chaleur et la tendresse correspondant à leurs besoins de caresses et d'amour.

Répondre aux besoins affectifs des bénéficiaires paraît difficile à réaliser et comporte des risques. Il s'agit de travailler, non plus uniquement sur des réactions physiologiques, mais sur du vécu émotionnel. Les membres du personnel doivent se surveiller pour ne pas céder à l'émotivité et à l'attachement amoureux. En premier lieu, l'employé doit se prémunir contre des réactions de pitié et de compassion trop 
intenses qui ne lui permettraient plus d'assumer, d'une manière efficace, ses tâches professionnelles ; il lui faut conserver une certaine insensibilité et contrôler ses émotions. En second lieu, il doit éviter qu'une relation amoureuse s'établisse entre lui et un patient. On dit de ce dernier qu'il est assoiffé d'affection et d'amour et qu'il peut rapidement s'amouracher d'un membre du personnel. L'employé doit rester lui-même et toujours répondre par un amour tendre et non passionné ; il est un ami et non un amant. La relation amoureuse entre un employé et un résident est perçue comme un amour impossible qui se termine dans le drame. $\grave{A}$ plusieurs reprises, on a évoqué l'histoire tragique d'un couple, une infirmière et un bénéficiaire, qui se sont donnés la mort. Ce récit illustre la fatalité attribuée au projet amoureux et cet événement dramatique, qui suscite la pitié et la terreur, sert de leçon.

C'est au modèle familial que réfère le personnel pour décrire les relations affectueuses entre les membres de la maisonnée hospitalière ; ces échanges pallient les aspects froids et impersonnels d'un hôpital. À la famille et à son caractère chaleureux et agréable, les personnes interviewées opposent la prison, le milieu hospitalier possédant certaines caractéristiques d'une maison de détention.

\section{L'hôpital : une prison}

Les propos des membres du personnel interviewés sur la condition de vie des bénéficiaires permettent de dégager une autre constante : être pris. Ces derniers sont pris par la maladie et pris en charge par un établissement hospitalier qui est souvent décrit comme une prison où les résidents doivent purger une peine (se purifier ou expier) : ils ont été pris en flagrant délit de maladie; ils sont condamnés à vivre dans un milieu institutionnel qui réglemente leur liberté d'action et de mouvement ; ils sont captifs du système hospitalier et subissent les contraintes de l'organisation des services d'un établissement de santé. Être enfermé et sortir accompagné sont les deux expressions utilisées pour exprimer les limites imposées à la liberté d'action du bénéficiaire.

La condition physique et, souvent, psychologique de celui-ci l'amène à être pris en charge par un centre hospitalier qui décide, pour lui, de ce qui est nécessaire à son bien-être. On lui demande de se soumettre au plan de services qui paraît le plus approprié à sa situation. Il devient ainsi subordonné à l'organisation hospitalière et, surtout, il devient un patient. En effet, on se le représente comme quelqu'un qui attend avec patience la venue d'une autre personne pour combler ses désirs. Pour certains professionnels interviewés, l'institution hospitalière agresse le patient et porte des coups à sa liberté et à son intégrité. On le dit victime 
d'une agression commise par le système hospitalier à son endroit. La passivité et la soumission qui lui sont imposées donnent l'impression qu'on lui reprend sa vie ; il peut être plongé dans un désarroi qui lui enlève le goût de vivre. La dégénérescence physique du résident et la routine aliénante de l'établissement le font mourir à petit feu et on rend l'institution coupable de cette disparition de la vie, de cet anéantissement.

Quelques professionnels étaient étonnés, voire indignés, de constater la soumission des bénéficiaires au mouvement mortifère de l'établissement. Il arrive, en effet, qu'on valorise l'obéissance et le conformisme chez les patients. Certains intervenants souhaiteraient pourtant que ceux-ci résistent à l'ordre institutionnel et déclarent publiquement leur opposition à la routine, leur refus de voir ces personnes être manipulées comme des enfants. Selon eux, ils devraient se prendre davantage en charge, définir eux-mêmes leurs besoins, sensibiliser les membres du personnel à leurs préoccupations et à leurs attentes: somme toute, prendre la défense de leur propre cause.

\section{L'ordre sexuel dans le milieu hospitalier}

Les membres du personnel décrivent donc le centre hospitalier sous deux angles: un milieu familial et un milieu carcéral. Ces deux représentations s'opposent, comme le chaud et le froid, l'affection et l'indifférence, le personnel et l'impersonnel. Par rapport à la sexualité, cependant, ces deux types d'institutions possèdent un dénominateur commun : elle est interdite et repoussée en périphérie.

\section{La sexualité : un sujet tabou}

Dans leur " maison ", les membres du personnel évitent de parler de sexualité avec les résidents : c'est un sujet tabou, une question personnelle qui doit demeurer secrète. Mieux vaut se taire parce qu'en parler, c'est la montrer, la faire exister. La dire, c'est la découvrir, la reconnaître, la faire. En gardant le silence sur le sexe, on l'ignore, on fait comme s'il n'existait pas.

On décrit les bénéficiaires comme des personnes qui s'engagent rarement dans des activités sexuelles. Ils limitent leurs gestes d'intimité à des expressions affectives élémentaires : se regarder, se prendre la main et donner un baiser. D'après certains professionnels, ces gestes leur paraissent suffisants et semblent les contenter. Pour d'autres, ce sont les seuls gestes qu'ils se permettent; ils ne font rien (de mal) 
d'autre. Cela leur donne un air innocent et de pureté (angélique) apprécié par certains et dénigré par d'autres parce que trop artificiel et limitatif. Somme toute, les gestes affectueux sont inoffensifs pour l'institution, ils ne nuisent pas à son bon fonctionnement, au contraire.

On cherche à garder le silence sur la sexualité; cette attitude correspond à la représentation que plusieurs se font de la sexualité des résidents : elle est absente (pas de réponse), éteinte ou en voie d'extinction chez les patients majoritairement vieux et malades. On se passe le mot pour ne pas troubler leur quiétude et leur calme. Il faut éviter de déclencher (ré-allumer) les désirs sexuels et d'inciter les bénéficiaires à y répondre. Chez les plus jeunes, la flamme pulsionnelle peut demeurer allumée et l'établissement doit voir à ce que les besoins sexuels soient satisfaits en tenant compte du contexte institutionnel. II n'est cependant pas facile d'identifier ces besoins et d'y répondre d'une manière adéquate et acceptable.

La sexualité des personnes hospitalisées est difficile à comprendre et à expliquer puisqu'elle demeure inconnue et secrète. Elles sont plutôt discrètes et évitent d'en parler. Deux facteurs sont souvent évoqués pour expliquer le silence et les inconnues à ce sujet: la dépendance des bénéficiaires, qui n'osent pas demander autre chose que ce qui est donné, et la mentalité conservatrice de la majorité d'entre eux qui entretiennent des sentiments de culpabilité et de honte, à cet égard. Ainsi patients et employés se retranchent dans leurs propres positions et maintiennent volontairement l'obscurité sur ce sujet. On prend des précautions pour dissimuler les besoins sexuels et en empêcher l'identification : ne pas en parler de part et d'autre.

Affirmer que l'établissement de santé est muet sur le sujet serait un témoignage de méconnaissance flagrante du milieu: ne pas en parler est un rêve, en parler beaucoup est la réalité. Les personnes interviewées ont indiqué qu'une des formes privilégiées pour en parler consistait à raconter des histoires comiques et à faire des farces. On cherche ainsi à se divertir et à mettre de la gaieté ; on aborde également, de façon directe, ce tabou : on parle de sexualité, on la déshabille, on la met à nu dans un milieu caractérisé par son asepsie sexuelle; c'est une façon de l'aborder, d'enfreindre un interdit sous une forme socialement acceptable. Les professionnels admettent que l'insistance mise à raconter des histoires de sexe traduit le malaise et la crainte qu'éprouvent les bénéficiaires à cet égard. L'analyse du contenu de ces histoires permettrait de préciser les préoccupations, les angoisses et les difficultés qu'ils rencontrent.

Outre les histoires, les rumeurs représentent une autre façon de parler de la sexualité. Il arrive souvent que des membres du personnel 
divulguent des événements qui devraient demeurés confidentiels. Le comportement sexuel des patients doit être gardé secret, mais tous ne savent pas le faire ; ils révèlent ce qui devrait rester caché ; ils cherchent à faire connaître et à rendre publiques des vérités qu'on préfère généralement ne pas ébruiter et colporter. En voulant se mettre au courant des derniers potins, on cherche bien plus qu'à faire la vérité sur des réalités mystérieuses: on veut savoir, non seulement pour mieux nommer mais aussi pour mieux normer. Faire du commérage apparaît comme un moyen de se défendre, de se réarmer collectivement pour faire face à la mise en question de l'ordre sexuel induite par certains comportements des résidents.

L'activité homosexuelle ou hétérosexuelle entre un(e) bénéficiaire et un(e) préposé(e) constitue une menace à l'identité et à l'ordre institutionnels. On les défend en racontant des histoires vécues ou imaginées qui amènent ceux qui parlent et écoutent à devenir des témoins de ce qui dérange, à analyser ce qui s'est passé et à se prononcer. Les histoires sont reprises, retravaillées, enrichies de multiples versions : elles tendent à devenir le support autonome des fantasmes collectifs à l'égard de la sexualité. Il en résulte souvent, pour ceux qui sont actifs sexuellement, un mélange de fascination et d'agressivité. Elles véhiculent d'autres normes (d'où leur attrait), mais elles désobéissent à une loi morale (d'où la nécessité de les corriger) prescrite par un pouvoir plus ou moins lointain, la direction ou le ministère des Affaires sociales. Souvent, répandre une nouvelle signifie rétablir l'ordre sexuel.

En plus des plaisanteries et des racontars, il y a les discussions sérieuses et les confidences. Ces dernières sont peu nombreuses mais certains professionnels indiquent qu'ils demeurent disponibles et acceptent d'en parler quand la question est abordée. Comme dans le modèle familial, les initiatives viennent des résidents et les membres du personnel sont prêts à les écouter. Quand on tente l'expérience, on découvre qu'il n'est pas facile de parler de la sexualité : c'est gênant, compliqué, embêtant... et c'est surtout épeurant. Il y a un danger que l'autre nous comprenne mal, soit affecté par nos propos, rapporte mal nos opinions ; on a peur d'être fautif. En parler ouvertement implique qu'on s'en mêle, qu'on s'explique, qu'on se justifie. On a parfois l'impression qu'on est le seul à le faire et que les autres ne sont pas tellement intéressés à discuter de cette question impossible et impensable. Alors on se tait nous aussi. 


\section{La sexualité mise au secret}

L'analyse des propos des employés a permis de mettre en évidence qu'il faut cacher le corps sexuel. On a décrit les sentiments de honte et de gêne qu'éprouvent des bénéficiaires à montrer leur corps nu. Cette réaction de pudeur est une réponse à l'intrusion du personnel dans les sphères d'intimité des résidents. Des gestes intimes, qui sont généralement reconnus comme des actes privés (par exemple se laver), deviennent des actes publics parce qu'ils sont pris en charge par les services de soins. Un certain tabou corporel est levé mais le tabou sexuel demeure. Les soins de santé obligent à se montrer nu mais le sexe doit demeurer caché. Les infirmier(e)s peuvent voir le corps nu du patient sans que ce dernier s'en offusque; ils ne sont pas des intrus mais des membres d'une même famille qui manipulent un être rendu docile. Le corps soigné peut être nu mais le corps libidinal doit être caché. Il est entré dans nos mours de réserver les gestes érotiques amoureux à des endroits privés, où la sexualité pourra librement s'exprimer. En public, il faut se retenir et ne pas attirer le regard par des gestes indécents. Voilà le dilemme : comment avoir une vie privée dans un milieu public?

Si un bénéficiaire désire satisfaire un besoin sexuel, il lui incombe de se trouver un endroit privé afin de ne pas s'exposer à la vue des autres. Il doit chercher un petit coin peu fréquenté où il pourra passer à l'acte sans déranger ni être dérangé. S'il a bien choisi son endroit et son moment, il ne risque pas de se faire prendre. Certains membres du personnel rencontrés croient qu'il est important d'accorder l'intimité nécessaire à la satisfaction des besoins sexuels. S'ils étaient à leur place, ils aimeraient ne pas être gênés et. embarrassés au cours de leurs activités sexuelles. Aussi appuient-ils l'initiative de ceux qui placent une carte avertissant les autres de ne pas les importuner puisque leur présence serait hors de propos.

Les personnes interviewées pensent que l'acte sexuel ne doit pas être montré. Les résidents doivent faire en sorte de ne pas être vus en action et se mettre à l'abri de tout regard. II arrive que les membres du personnel leur demandent de faire attention parce qu'il y a un danger trop évident d'être vus et d'être pris. L'importance de cacher la sexualité vient surtout du fait que certaines personnes de l'entourage ne veulent rien savoir des activités sexuelles. Elles ne veulent pas les constater, les reconnaître et avoir à prendre position.

La présence et la visite de personnes âgées obligent les employés à dissimuler les évidences puisque ces dernières seraient offusquées et même scandalisées de découvrir un bénéficiaire en flagrant délit d'activités sexuelles. Ce n'est pas seulement choquant; c'est menaçant puisque la 
réputation de l'établissement est en jeu. Un scandale mettant en cause cette question peut compromettre et ternir l'image de l'institution et ceux qui y travaillent. En réprimant la sexualité, c'est le caractère irréprochable des mœurs du centre hospitalier que l'on veut sauvegarder ; on cherche aussi à maintenir la considération et l'estime que les autres ont pour soi et qu'on a pour soi-même.

\section{La politique sexuelle cachée}

Face à une situation problématique, quelle ligne de conduite doiton adopter? Dans ce domaine, il ne semble pas exister de politique formelle. Les membres du personnel n'ont pas reçu d'instructions particulières sur ce qu'ils doivent faire lors d'événements à caractère sexuel. Ils ne connaissent pas les idées de la direction de l'établissement sur cette question et ne peuvent donc pas se situer par rapport aux visions des dirigeants. La politique concernant la sexualité paraît aussi secrète et cachée que cette dernière. Peut-il y en avoir une au sujet d'une réalité qu'on aimerait voir disparaître? II n'y a peut-être pas de normes et de règles formelles, mais il existe des croyances et des pratiques qui sont transmises par la parole et l'exemple. Ainsi, les employés doivent intervenir dans certaines situations (cas d'espèce) et leurs actions expriment la façon naturelle et normale d'agir quand ces événements se présentent (cas types). Les façons de penser et d'agir face à l'expression de la sexualité sont consacrées par la tradition hospitalière. Ainsi, les orientations n'ont plus besoin d'être affichées; elles se trouvent dans la tête de chacun sous forme de représentatiaons intériorisées.

Est-ce correct? et Que fait-on? sont deux questions qui réfèrent l'une à l'évaluation, l'autre à la décision. Certaines situations à caractère sexuel obligent les membres du personnel à s'interroger afin de déterminer si elles sont acceptables et quelles réponses seraient les plus appropriées. Il faut alors tracer une ligne de démarcation entre ce qui est correct et ce qui ne l'est pas. Selon les témoignages des personnes interviewées, ce sont surtout des critères institutionnels qui permettent de faire cette discrimination. La sexualité est, non pas acceptée, mais tolérée en autant qu'elle ne dérange pas le bon fonctionnement de l'établissement et n'a pas de conséquences fâcheuses ; mais on pourrait éventuellement blâmer la personne pour cette action. Un comportement sexuel peut aller trop loin et dépasser le seuil d'acceptabilité ; alors ce geste troublant perturbe l'ordre social et interrompt la tranquillité du milieu. Certains membres du personnel croient qu'il est déplacé pour un bénéficiaire de recevoir des visites " arnicales " à des heures indues ; 
dans ces situations, ce n'est pas la rencontre sexuelle qui est visée mais plutôt la conduite déréglée, la manifestation d'indiscipline qui sème le désordre.

II arrive que des patients touchent et caressent des employées. Ces gestes sont jugés anodins lorsqu'ils sont perçus comme des marques d'affection ou un jeu. Par contre, si on devine une intention érotique, ou considère qu'il y a manquement aux convenances et on montre alors sa désapprobation en évitant le toucher ou, pour certaines, en remettant le "coupable " à sa place. Celui-ci, en faisant un toucher érotique, ne respecte pas le pacte de neutralité qui fait partie du contrat de services professionnels. L'employée est là pour s'occuper de son bien-être et lui demande de la considérer comme une professionnelle non éligible sexuellement. Sinon, le bénéficiaire est repris et même réprimandé puisqu'il dérange ; on le rappelle à l'ordre.

Avec certains résidents qui font des gestes inconvenants, les employés sont sur leurs gardes. On n'a pas encore réussi à les mettre à leur place. Ils sont difficilement récupérables. Alors on les a à l'œil et on informe les autres de leurs manies. Il arrive même qu'on les réprimande et qu'on les punisse. On cherche ainsi à les empêcher d'exprimer une pulsion condamnable et, surtout, dangereuse pour l'établissement.

\section{Le tabou d'incentre}

L'objectif principal de l'étude consistait à dégager, par le biais des représentations des membres du personnel, la politique relative à la vie institutionnelle et à la sexualité. Quelle est cette politique? L'analyse des discours a permis d'en dégager le principe directeur : la sexualité est défendue, ce qui se traduit par la valorisation du silence et de l'abstinence. Il faut dissimuler les activités sexuelles, se taire et les cacher.

Cette dévalorisation n'est pas particulière au centre hospitalier qui fut étudié. D'autres recherches traitant de ce phénomène chez des personnes âgées vivant en institution ont dégagé ce même constat (Kass, 1978 ; Paradowski, 1977 ; Schlesinger, 1983). L'étude de la politique institutionnelle nous renvoie au cour de notre problématique: "Pourquoi interdire la sexualité en institution hospitalière? ". II nous paraît trop facile et trop simple d'accuser les employés d'un trop grand puritanisme. Ce refus d'accuser des personnes est bien plus qu'une simple attitude bienveillante à leur endroit ; elle correspond, de fait, à une démarche analytique qui consiste à dégager les composantes et les enjeux psychosociaux de l'interdit sexuel en institution. Nous pensons 
que les membres du personnel n'ont pas la pleine conscience ni la pleine maîtrise de la politique sexuelle de l'établissement puisque sa structuration et ses effets prennent place à des niveaux qui échappent souvent au savoir et au vouloir des personnes impliquées. Dans cette perspective, notre analyse portera sur les rapports entre la sexualité et I'institution hospitalière qui, maintes fois, sont du domaine du non-dit et du non-su. C'est à partir du concept central de tabou d'incentre (interdit sexuel dans les centres de santé) que nous essayerons d'ordonner et de comprendre les discours présentés par les professionnels.

Dans leurs propos, nous avons dégagé un sentiment de peur à l'égard de la sexualité. Cette sexophobie se traduit par une crainte pour leur intégrité professionnelle et la réputation du centre hospitalier. Soulignons d'abord les risques associés à l'exercice de leur profession. Lors de leurs activités quotidiennes, les intervenants peuvent observer des manifestations sexuelles et même y être impliqués. Il leur arrive aussi d'entendre des conversations, d'être interpellés, et d'être invités à avoir des rapports intimes comme si ceux-ci avaient le pouvoir de raccorder la vie privée et la vie professionnelle. Ces approches entre patients et employés représentent des dérogations à la neutralité professionnelle : il y a un risque de confusion et de mélange qui constitue une atteinte à l'identité de chacun et à l'ordre institutionnel ; et il y a un risque de ternir la réputation de l'établissement. Si les activités sexuelles sont mal contrôlées, les visiteurs peuvent reprocher aux intervenants de ne pas prendre les mesures nécessaires pour les contenir. Elles peuvent contribuer à remettre en cause la compétence de l'établissement et de son personnel à bien réaliser sa mission. Dans cette perspective, il nous est possible de comprendre la raison de l'existence du tabou sexuel au sein du centre hospitalier.

La sexualité est refusée et cachée parce qu'elle est impure. Dans son étude sur le tabou, Cazeneuve (1971) affirme qu' "Est impur ce qui empêche le groupe social de gratifier tous ses membres d'une existence quiète, sans angoisse, sans problème individuel, sans imprévu » (p. 54). Au centre hospitalier, la sexualité est impure du fait qu'elle éveille chez le personnel un sentiment d'angoisse devant les dangers qu'elle peut provoquer. Pour se défendre et conjurer cette menace, il est naturel qu'on essaie de la tenir à l'écart par des interdictions. Sa libre expression risque d'engendrer un dérèglement individuel et collectif craint au plus haut point par l'établissement. L'interdit sexuel représente une mesure défensive pour empêcher que le malheur arrive.

L'ordre peut être perturbé par la confusion engendrée par les rapports sexuels entre les bénéficiaires et le personnel. Le récit d'une relation «impossible» entre un patient et une infirmière illustre ce fait. 
On juge cette liaison inacceptable puisqu'elle réconcilie des éléments séparés. Dans un établissement stable et bien réglé, chaque personne et chaque chose est à sa place ; chaque catégorie d'êtres et d'objets est bien distinguée des autres. Une liaison amoureuse bénéficiaire-infirmière représente une dérogation à un système de règles en vigueur et menace l'équilibre institutionnel : aussi, son récit joue le rôle de " porte-malheur ". L'histoire se termine par le suicide du couple interdit. Dans ce contexte, le tabou d'incentre est un porte-bonheur du fait qu'il protège les résidents et le personnel contre le désordre issu de l'impureté.

Le tabou d'incentre constitue l'interdiction de bouleverser l'ordre institutionnel du milieu hospitalier. La sexualité suscite une angoisse profonde puisqu'elle symbolise l'instabilité et le déséquilibre. Elle risque de troubler la tranquillité recherchée par le personnel. Elle ne suscite pas seulement la crainte, mais également l'aversion. La personne qui transgresse l'interdit sexuel doit être dénoncée et purifiée avant qu'elle ne contamine d'autres personnes. Ainsi, certains membres du personnel jugent important de dépister l'impureté pour la rapporter aux autorités. La répugnance associée à la découverte du délit sexuel est moins un dégoût à l'égard de l'activité sexuelle en elle-même qu'une répulsion face à l'impureté, à la transgression du tabou. L'individu impur soulève l'indignation et la réprobation puisque son geste perturbe l'équilibre institutionnel. Il doit subir les conséquences directes de sa faute (par exemple : étiquetage, isolement, expulsion) ; il peut se racheter par un rite de purification (par exemple : aveu, regret, punition). Ces mécanismes de contrôle s'inscrivent dans l'intérêt que porte l'établissement à faire respecter le tabou d'incentre.

Si la sexualité évoque l'impureté, l'affectivité est plutôt associée avec la pureté. On interdit la première, on encourage la seconde. Les bénéficiaires sont déracinés de leur milieu d'origine et placés en institution hospitalière. Pour éviter leur régression psychologique, le personnel s'efforce de créer un climat chaleureux. L'affection est une force positive qui conserve la vie et donne la santé et ses manifestations apparaissent empreintes d'un caractère salutaire qui inspire la confiance et la vénération. L'affectivité s'oppose à la sexualité du fait que cette dernière est interdite et condamnée. L'une rend solide et sain, stable et régulier, l'autre, faible et malade, instable et irrégulier. Les énergies vivantes (l'affect) et les forces de mort (le sexe) se rassemblent pour former les pôles attractif et répulsif. L'affection assure la cohésion; la sexualité engendre la dissolution.

L'attribution d'un statut de " famille " à l'établissement vient renforcer l'importance de l'affection, et par le fait même le tabou d'incentre. Le cadre familial est un modèle de référence fortement valorisé par le 
personnel. L'unité hospitalière vient remplacer, pour les bénéficiaires, I'unité familiale qu'ils ont dû quitter. Les relations et les échanges, dans le cadre des activités quotidiennes de l'hôpital, vont faire naître une parenté " sociale ". On recherche un contexte familial puisque la famille est reconnue pour ses fonctions de sauvegarde corporelle, de sécurité affective et de protection. Dans le milieu hospitalier, le patient peut s'attendre à trouver soins, chaleur, réconfort et compréhension. Le recours à l'image familiale permet de rasséréner le milieu hospitalier, en donnant l'impression d'évacuer les tensions et les déviations. L'idée d'une "atmosphère familiale" évoque le calme, la sérénité et le réconfort.

Il est difficile pour le personnel de verbaliser ce qu'il entend par climat familial. Jusqu'à un certain point, chaque unité a son style familial, compte tenu des personnes qui la dirigent, y travaillent et y sont accueillies. Il devient alors difficile de transcrire dans les textes une politique familiale ; il est plus facile de la vivre que de l'écrire. Pour la décrire, il faut référer aux valeurs abstraites d'un modèle familial général et de ses vertus incantatoires; ainsi la famille permet de satisfaire les besoins de subsistance, de soins, d'appartenance et de relations intimes. Pour ce faire, elle doit valoriser la stabilité, la cohésion, l'attachement et autres qualités " familiales ». Dans le but de consolider le modèle familial, les gestes affectueux seront très valorisés dans l'établissement de soins prolongés. Source bienfaisante de chaleur, il devient un foyer familial où il fait bon vivre en harmonie les uns près des autres; il devient aussi un refuge ou l'on trouve réconfort contre la maladie et la solitude. L'image de maison familiale est valorisée du fait que l'hôpital vient conjurer les peurs d'abandon et de mort chez des personnes sérieusement malades.

Le climat familial de l'établissement hospitalier vient donner un sens à l'interdit sexuel. Les personnes vivant et travaillant à l'hôpital appartiennent au même groupe familial. D'une manière symbolique, elles sont parentes, donc sujettes à interdiction. Il se peut fort bien que l'observation ou la participation à des activités sexuelles dans l'établissement suscite une angoisse semblable à celle observée pour l'acte incestueux. Pour les bénéficiaires, le personnel pourrait jouer le rôle de substitut familial (fils-fille ou frère-sœur). De même pour l'employé, le bénéficiaire symbolise un père ou un frère. Ainsi le tabou d'incentre est renforcé par l'interdiction de relations sexuelles entre les membres d'une même famille, représentée ici par le milieu hospitalier. D'une manière plus ou moins consciente, les uns et les autres gardent leurs distances et évitent une trop grande intimité afin d'éviter l'acte " incentrueux ». Ce dernier serait la réalisation d'un désir érotique qui viendrait 
perturber émotivement les individus et, socialement, l'ordre institutionnel. Le tabou d'incentre permet d'éviter ces conséquences néfastes.

La présente explication de l'interdit sexuel en institution donne un nouveau sens à certains gestes sexuels posés par les résidents à l'égard du personnel féminin. Pour s'amuser et pour les taquiner, les bénéficiaires font des touchers défendus. L'infirmière représente à leurs yeux un être tabou qu'il est interdit de toucher d'une manière érotique. II est dangereux de faire ce geste, ce qui vivifie l'attrait sexuel pour cette personne interdite et, par le fait même, amplifie le désir de poser ce geste « incentrueux ». En disant qu'il aimerait le faire ou en le faisant, il revit ses sentiments ambivalents à l'égard de l'être tabou : l'aversion et l'attraction. Le plus souvent, l'enjeu de ce geste est inéluctable: le bénéficiaire s'attend à se faire mettre à sa place. On lui demande de respecter le tabou sexuel et de s'abstenir ou de réserver ce geste pour les personnes accessibles (par exemple: son amie ou son épouse).

\section{Conclusion}

Le tabou d'incentre apparaît comme un interdit obligatoire qui rend de multiples services sur les plans individuel et social. La prohibition des activités sexuelles au sein de l'établissement hospitalier correspondrait à un besoin impérieux de sauvegarder l'intégrité physique et morale des personnes qui y séjournent ou y travaillent. La libérer de l'interdit exigerait une transformation radicale des mentalités et des pratiques au sein de l'établissement. Il faudrait révolutionner la conception de l'hôpital, de la maladie, de la mort et de la sexualité. Ce projet est séduisant mais manque de réalisme. Dans les institutions, les mutations se font avec moins d'éclat et plus lentement puisque les enjeux sont multiples et complexes. Le processus de modernisation qui anime, depuis un certain temps, les établissements de santé témoigne toutefois d'une volonté d'assurer une meilleure qualité de vie sexuelle chez les bénéficiaires.

L'analyse des discours a révélé une première voie qui mène au changement. Parler de la sexualité, c'est aussi faire quelque chose pour la changer et l'améliorer : ce n'est pas seulement refléter et exprimer nos désirs, nos plaisirs et nos insatisfactions ; cela signifie aussi remettre en question les habitudes et s'ouvrir à d'autres perspectives. II est important que les soignants et les soignés se posent et posent des questions sur ce sujet. Ils doivent se permettre d'exprimer des interrogations maintes fois ignorées ou refoulées. Le plus souvent, la parole est dévalorisée puisqu'elle paraît inefficace face à des moyens techniques 
indiscutables. Toutefois, elle possède une valeur reconstructive car elle élucide les raisons qui sous-tendent les interrogations, même si on n'y trouve pas immédiatement de réponse. Elle permet aussi de mieux comprendre les difficultés à assumer sa sexualité et à entrevoir les obstacles qui entravent cette intégration. La recherche de solutions sécurisantes cède la place à une réflexion plurielle où l'on découvre ses compétences et ses limites à se prendre en charge et à aider l'autre à le faire.

Les intervenants et les bénéficiaires demandent, qu'au cours des périodes d'échange, les discussions soient simples et pratiques. II est difficile de résister au piège d'un rationalisme technique qui ne reconnaît que les actions considérées comme efficaces et utiles. Les conseils et les recettes dépossèdent les soignants et les soignés de leur parole au profit d'une ligne de conduite présentée comme irréfutable et infaillible. C'est aussi l'angoisse devant la sexualité et la mort qu'on essaie de tromper. On doit donc éviter de leur dire ce qu'ils doivent faire mais plutôt les aider à dire eux-mêmes ce qu'ils vivent et veulent vivre. Les angoisses, les frustrations, les espoirs ne sont pas niés et retenus. Les positions individuelle et institutionnelle de repli et d'oubli n'ont plus de sens là où les réponses à la peur doivent conduire à une nouvelle solidarité collective.

Ce projet incite les employés et les patients, non pas à couvrir mais à découvrir des états de choses et de vie, comme les inconforts et les insatisfactions face à la sexualité dont il ne faut pas ou ne faudrait pas parler, dont il n'est pas permis de parler. Il ne s'agit pas de dénoncer des activités sexuelles illicites mais d'énoncer les inquiétudes à l'égard de ces pratiques. II ne s'agit pas non plus de prouver qu'on a raison et que l'autre a tort mais d'éprouver ses contradictions et ses conflits. II faut rendre possible une libération de paroles; ainsi on arrive à montrer qu'en parlant de la sexualité on ne partage pas seulement un secret, un problème personnel, mais le secret qu'on doit taire, le problème dont personne ne doit parler. Lors des réunions, il faut éviter de tout dire et de tout avouer. En forçant la personne à parler et à tout divulguer, on tombe dans le piège des rumeurs et, pis encore, on instaure un nouveau mécanisme de contrôle par l'aveu. Les réunions doivent plutôt permettre une ouverture d'esprit et une ouverture à la transformation par des échanges propices à une réappropriation de son vécu et de ses aspirations par rapport à la sexualité. En en parlant, on découvre aussi qu'il est possible de transformer les conditions de vie dans l'établissement. La parole se prolonge dans des actions concrètes pour améliorer le bienêtre sexuel des bénéficiaires.

Si les établissements de santé cherchent vraiment à procurer à leurs résidents une bonne santé sexuelle, il est essentiel de clarifier le statut 
ambigu relatif à cette question et de faire reconnaître sa richesse. Des personnes interviewées ont indiqué que l'activité sexuelle entraînait des problèmes mais qu'elle pourrait aussi être bénéfique, car elle possède une force qui peut apporter des biens et des maux, suivant les circonstances particulières de ses manifestations. Elle est virtuellement ambiguë ; elle est une source de bénédictions ou un foyer de malédictions. La politique des établissements de santé devra être guidée par une conception positive de la sexualité et sa philosophie et les actions qu'elle propose devront en faire valoir les bénéfices. Elle doit être présentée et recherchée comme une force positive qui conserve la vie et donne la santé et elle doit inspirer la confiance et la considération; ainsi, au lieu de provoquer la peur, elle serait une source d'espoir.

\section{Note}

1 L'étude des propos des bénéficiaires est présentée dans un autre article (Dupras, 1986).

\section{Références bibliographiques}

Cazeneuve, J., Sociologie du rite : tabou, magie, sacré, Paris, Presses Universitaires de France, 1971.

Corneluus, D. et al., Who Cares? A Handbook on Sex Education for the Physically Disabled, Baltimore, University Park Press, 1979.

Dupras, A., J.J. LÉvy et M.A. AuclaIr (éds), Sexualité et difficultés d'adaptation, Montréal, Éditions IRIS, 1984.

DUPRAS, A., "La peur de la sexualité chez des bénéficiaires âgés résidant dans un centre hospitalier de soins prolongés", 1986, (à paraître).

HALL, J.E. et H.W. SAWYER, "Sexual policies for the mentally retarded", Sexuality and Disability, vol. 1, no 1, $1978: 34-43$.

KASS, M.J., "Sexual expression of the elderly in nursing homes ", The Gerontologist, vol. 18, no 4, 1978 : 372-378.

Keitner, G. et P. Grof, "Sexual and emotional intimacy between psychiatric inpatients: formulating a policy", Hospital and Community Psychiatry, vol. 32, no 3, 1981 : 188-193.

PARADOWSKI, W., "Socialization patterns and sexual problems of the institutionalized chronically ill and physically disabled ", Archives of Physical Medecine and Rehabilitation, vol. 58, no 2, 1977 : 53-59.

SCHLESINGER, B., "Institutional life: The canadian experience", R.B. WEG (éd.), Sexuality in the Later Years, New York, Academic Press, 1983 : 259-269. 
SolNIK, R.L. (éd.), Sexuality and Aging, Los Angeles, University of Southern California, 1978.

STRIAR, S. et al., "Guidelines for responding to residents' social-sexual behaviors ", dans: Policy and Procedure Manual, Ann Arbor (Mich.), Plymouth Center for Human Development, 1981.

SZASZ, G., "Sexual incidents in an extended care unit for aged men ", Journal of the American Geriatrics Society, vol. 31, no 7, 1983 : 407-411.

WEG, R.B. (éd.), Sexuality in the Later Years, New York, Academic Press, 1983. 